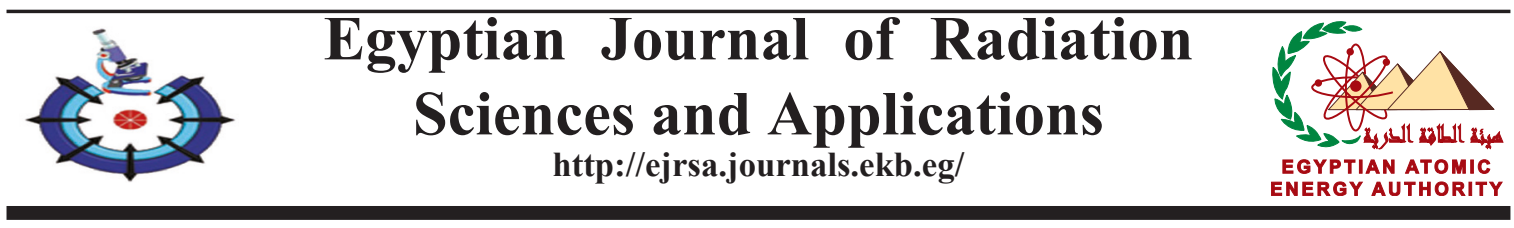

\title{
Performance Assessment of NaI Detector Spectrometer for Safeguard Measurements
}

R.A. El-Tayebany", M.H. Hazzaa, S.E.Shaban

Egyptian Nuclear and Radiological Regulatory Authority (ENRRA), Cairo, Egypt.

\begin{abstract}
$\mathbf{N}$ UCLEAR Safeguards Measurement Methods are developed by design characteristics as well as performance specifications for several systems of the gamma-ray assay. $\mathrm{A} \mathrm{NaI}(\mathrm{Tl})$ gamma detector was characterized with respect to count rate performance, detector efficiency, dead time (DT), low-level discriminator, and threshold effects using low, medium, and highenergy radioactive point sources. Spectral data are collected using inorganic scintillation detectors $\mathrm{NaI}(\mathrm{Tl})$. Besides, the response functions of the $\mathrm{NaI}(\mathrm{T} 1) 2 " \times 2$ " detector were determined where detector efficiency and FWHM were measured at diverse sources using energy lines (356.02 keV of 133Ba, $661.7 \mathrm{keV}$ of $137 \mathrm{Cs}$ and1173.2 and $1332.5 \mathrm{keV}$ of $60 \mathrm{Co}$ ) and calculated by using MCNP method. The Gaussian spectra generated by MCNP-5 code were compared with the experimental work. To validate the simulated result, the Gaussian Energy Broadening (GEB) card was used, yielding the best results. When comparing the obtained findings derived from MNCP calculations with the experimental findings, they showed a good agreement in their values.
\end{abstract}

Keywords: Dead time (DT), Low- level discriminator (LLD), NaI(Tl) detector Efficiency, Non- destructive assay techniques (NDA).

Gamma-ray spectroscopy is commonly employed in nondestructive assay techniques (NDA). A central aspect of accurate quantification of nuclear material (NM) is the efficiency calibration of a given NDA gamma system. The resultant count rate in the gamma spectrum is dependent on the detector efficiency, the number of events recorded by the system per gamma-ray emitted, the gamma-ray energy, the geometry of the entire setup, and the composition of any material in the path of the detector (Savidou et al., 2008; Nakazawa et al., 2010; Unz et al., 2011).

Scintillation detectors are widely used in NDA laboratories due to their good resolution, high efficiency, and moderate energy resolution. In many cases, MCA is also provided with a single channel analyzer (SCA). Parallel to the SCA, the input pulses provide the equivalent amplitude requirements established by the SCA. Such a pulse is passed through MCA circuitry. Such a step aims to permit the refusal of smaller or larger input pulses than SCA-limited area of interest. Such limits, usually known as lowerlevel discriminator (LLD) as well as upper level discriminator (ULD), are selected for exclusion of very small as well as extremely high noise pulses at lower and upper ends outside spectrum of interest, respectively (Glenn \& Knoll, 2010). Therefore, such uninteresting pulses never reach the Analoge to Digital Converter (ADC) and as a result they are not using significant conversion time, instead the fractional dead time will increase. Thus, it is highly recommended to greatly minimize the counting rate provided to the input gate through exclusion of noise as well as any insignificant small-amplitude events with LLD, and when substantial numbers of large-

\#Corresponding author email: reltyebany@ymail.com

Received 23/8/2020; Accepted 14/9/2020

DOI: $10.21608 /$ ejrsa.2020.40247.1103

C2020 National Information and Documentation Center (NIDOC) 
amplitude background events are available to be omitted with an acceptable ULD configuration.

In the case of isolation of a radioactive source as well as scintillation detector from all encircling materials, the detected pulse-amplitude spectrum in terms of shape and magnitude would depend solely on the gamma-ray energy, the physically inherent characteristics of both source and detector in addition to the geometrical connection between both of them. Practically, several variables linked to the experimental setting would influence the detected pulse-amplitude distribution pattern (Heath, 1997; Lodge et al., 2015).

$\mathrm{NaI}(\mathrm{Tl})$, as well as HPGe detectors, are the most common detection systems dedicated to $\gamma$-spectrometry (Ahmadi et al., 2009). Among several features, the system efficiency is a highly significant characteristic possessed by a detector. For instance, the $\mathrm{NaI}(\mathrm{Tl})$ detector demonstrated substantial merit in terms of high detection efficiency (Kaneko et al., 1998).

A powerful Monte Carlo computer program has been defined to simulate and measure total and full-energy peak (absolute and intrinsic) efficiency of the cylindrical $\mathrm{NaI}(\mathrm{Tl})$ detector (Kadhem \& Mohammed, 2010). The findings indicate that the experimental results and other error rate estimates are very acceptable at a rate of less than $2 \%$, and the findings can be utilized in $\gamma$ - spectroscopy and in sources activity determination.

Since many years ago, the increasingly popular MC simulation technique (Ewa et al., 2001; Lepy et al., 2001; Karamanis, 2003) was employed by several authors for simulating the gamma-ray detection process. The technique was exploited for calculating the response traits of diverse types of germanium detectors at mono-energetic as well as diverse ranges of gamma-ray (Wainio et al., 1966; De Castro et al., 1967; Meixne, 1974; Avignone, 1980; Varley et al., 1981; Michel et al., 1986; Fehrenbacher et al., 1996; Hurtado et al., 2004a; Salgado et al., 2006). Besides, MC was also utilized to calibrate detectors effectively, either by a direct method or in combination with other experimental measurements (Debertin \& Grosswendt, 1982; Ashrfi et al., 1999; Laborie et al., 2000; Rodenas et al., 2000; Saegusa et al., 2000; Ewa et al., 2001; Kamboj \& Kahn, 2003; Karamanis, 2003; Arnold \& Sima, 2004; Hurtado et al., 2004b; El-Gammal et al., 2005).

$$
\text { Gamma ray spectrometry (NaI) is }
$$

characterized in this work for safeguards application. The manufacturer data and other data from $\mathrm{X}$ ray imaging are used to characterize and construct a detector model in conjunction with MCNP code. The Monte Carlo method was used to calculate the photon detection efficiency for a 3"X3" NaI detector exposed to gamma ray at energy of $661.7 \mathrm{keV}$ at various distances for validation of the detector model. The results that have been obtained by MCNP detector model are compared with the experimental results. An agreement was found between calculated and experimental results of detector efficiency with accuracy ranging from $-1.02947 \%$ to $1.49405 \%$, which confirms the validity of the detector mode (Ahmed, 2017).

Comparative studies of absolute efficiency and ${ }^{235} \mathrm{U}$ content in samples using different detectors are useless unless the detector specifications are known. This study attempts to overcome the difficulties facing MCNP code related to geometry by the aid of ISOCS software. Also, it overcomes the issue of comparing absolute efficiency outcomes from detectors of different geometry using a mathematical model and measured standards. The results of measured count rates, absolute efficiency and ${ }^{235} \mathrm{U}$ mass for five uranium oxide $\left(\mathrm{U}_{3} \mathrm{O}_{8}\right)$ standards that have different enrichments were used to perform a correlation between different gamma detectors (Abdel-Monem et al., 2019).

The purpose of this study is to evaluate the performance of the gamma spectrometer based on $\mathrm{NaI}$ detector for safeguard measurements. This can be done by studying the response function of $\mathrm{NaI}$ detector at a wide range of energy scale, as well as overcoming the excluding noise and other insignificant events by varying the LLD and threshold values that contribute to improving the performance of the measurement level in safeguards field.

\section{Materials and Methods}

Radiation sources $\left({ }^{133} \mathrm{Ba},{ }^{137} \mathrm{Cs}\right.$ and $\left.{ }^{60} \mathrm{Co}\right)$ that give $365.02,661.7,1173.2$, and $1332.5 \mathrm{keV}$ of gamma-ray energy, respectively, were positioned distant from the detector face by $0,2,4,6,8$ and $10 \mathrm{~cm}$. Each measurement was carried out over $1000 \mathrm{sec}$ for realizing good statistics to evaluate each gamma peak. For all utilized radionuclides in the current research, the datasheet of photon energy values and activity values was listed, as shown in Table 1. 
TABLE 1. The used Gamma Reference Point Sources (Eckert \& Ziegler, 2007).

\begin{tabular}{lccc}
\hline Radioactive source & Activity $(\mathbf{k B q})$ & Uncertainty & Energy lines $(\mathbf{k e V})$ \\
\hline${ }^{133} \mathrm{Ba}$ & 37.8 & $5 \%$ & 356.017 \\
${ }^{137} \mathrm{Cs}$ & 185.1 & $3 \%$ & 661.657 \\
${ }^{60} \mathrm{Co}$ & 163.9 & $3 \%$ & $1173.237,1332.5$ \\
\hline
\end{tabular}

In the present work, the utilized gamma-ray spectrometer is regarded as a portable scintillation detector assembly on the basis of a Miniature Multi-Channel Analyzer model (MCA-166), featuring a $\mathrm{NaI}(\mathrm{Tl})$ detector Crismates model, and operated at $+600 \mathrm{~V}$, with relative efficiency $3.297 \times 10^{-5}$. The detector, as supplied by the manufacturer, possesses a $\mathrm{NaI}(\mathrm{Tl})$ crystal having dimensions of $5.08 \times 5.08 \mathrm{~mm}$ as well as $1 \mathrm{~mm}$ thick aluminum housing. In experimental work, the source is placed in front of the detector and close to the Al- cap of the detector. Figure 1 displays the experimental setup employed in the present work.

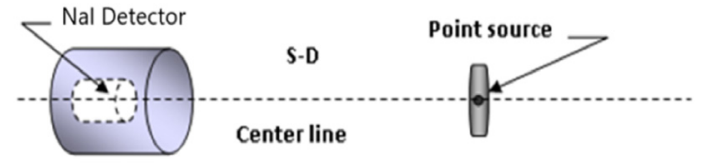

Fig. 1. Set up configuration using NaI detector and point source.

\section{MCNP calculations}

The MCNP is an advanced MC simulation program that provides all required crosssectional data for neutron, photon as well as electron transport measurements (X-5 Monte Carlo Team, 2003). MC simulation is considered to be a random numbers-sequence that occurs throughout the simulation. The simulation, based on repeating this sequence, will yield results in agreement with the obtained ones from the first sequence (Landau \& Binder, 2009). Computing the absolute detector efficiency was achieved using the general Monte Carlo code (MCNP5). In order to simulate the experimental setup and according to available details, modeling of both specifications and characteristics of the NaI detector as well as utilized point sources was accomplished. The GEB input card (Gaussian energy broadening) in MCNP-5 was utilized for the simulation of peak height uncertainty. GEB card coefficients were determined, as guided in the MCNP5-manual, through FWHM-value measurement at different energies in spectra determined under different count rates (X-5 Monte Carlo Team, 2003).

To run this calculation, 20 input created files are required, which use $10^{8}$ histories (number of photons) with a run time of 20 minutes. The employed laptop has specifications of $2.5 \mathrm{GHz}$ Intel Core i5 processor, where the detector pulse height can be determined by tally F8. The absolute detector efficiency was calculated by F8 at different energy lines.

\section{Results and Discussion}

\section{Energy resolution}

This work was carried out by modeling and validating the $\mathrm{NaI}(\mathrm{Tl})$ detector for gamma-ray energy using standard gamma-ray sources placed close to the end cap detector. The experimental work is optimized by testing the full output spectrum at different source to detector (SD) distances from $(0-10) \mathrm{cm}$ to reduce the background and dead time as low as possible. From the mesurments, the (S-D) distance $=6 \mathrm{~cm}$ is selected to be the optimum distance where the dead time $<3 \%$.

The relation between the square root of energy lines and FWHM gave fitting linear relation as shown in Fig. 2. The constants produced from the fitting equation were used in the GEB card in the MCNP input file to obtain matching in full energy beak broadening between the experimental and calculated results. The comparison for the results of the response function at $661.7 \mathrm{keV}$ for ${ }^{137} \mathrm{Cs}$ for broadening using GEB card, and non- broadening cases in Monte Carlo code is shown in Fig. 3. This indicates that the experimental result of the response function of the $\mathrm{NaI}(\mathrm{Tl})$ detector is in excellent agreement with the Monte Carlo prediction using the GEB card. Where the energy regions of interest (ROIs) used for the detectors were selected to minimize interference. The gamma energies used are $(356.017,661.7$, 1173.5 , and $1333.5 \mathrm{keV}$ for ${ }^{133} \mathrm{Ba},{ }^{137} \mathrm{Cs}$ and $\left.{ }^{60} \mathrm{Co}\right)$. 


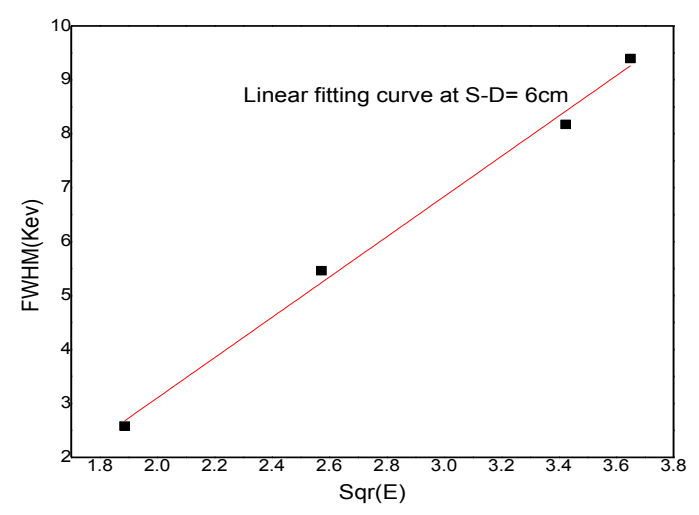

Fig. 2. Linear fitting of $\sqrt{ } \mathrm{E}$ versus FWHM.

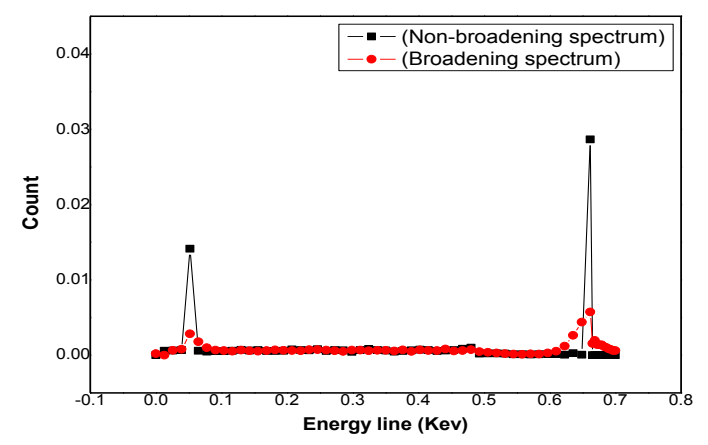

Fig. 3. Response function at $661.7 \mathrm{kev}$ for ${ }^{137} \mathrm{Cs}$ with using MCNP-5 code.

\section{Efficiency}

The detector efficiency is considered the most crucial factor for response function determination. It strongly depends on the parameters such as the energy of gamma rays, detector dimensions, source dimensions, geometric arrangement of the detector and source, and density of the sample (Tarim \& Gurler, 2018). The effect of source-todetector distance and energy on the full energy peak and total efficiencies for the studied gamma ray energies are presented as shown in Fig. 4. The photon detection efficiency was also used for the validation of the developed model. The efficiency was obtained for four different gamma ray energies at different positions (source-todetector distance) values. It can be seen that the detection efficiency has decreased with increasing the S-D distance. Adjustment of(S-D) distance is the main factor for setup optimization. Different values of distances are tested to reach the optimum position, at which the dead time and background are as low as possible, for the standard sources with respect to the detector face.

\section{Low level discriminator}

So many typical characteristics show up on most ADCs, regardless of the type or the manufacturer. LLD, as well as ULD, decide the smallest and largest pulses validated for digitization, respectively. The discriminators can be configured for rejecting uninteresting low- as well as high-energy events and reducing the ADC dead time. When adjusted, the discriminator does not influence the overall count rate of gammaray and it is unable to be utilized for reducing pulse pileup losses occurring in the detector, preamplifier, as well as amplifier (Doug et al., 1991).

Figure 5 displays the influence of LLD on the net area of the interested energy lines where all the used point sources are measured at the same time. Consequently, the spectrum includes all energy lines emitted from all the used point sources.

In Fig. 5, there are differences in the recorded net area which changed due to changes in the LLD values. The Figure shows that the fair stability of the net area values of the interested photo peak are at energy lines of 356.017, 661.7.1173.2 and $1332.5 \mathrm{keV}$ starting from $\mathrm{LLD}=4$ to 10 .

The effect of $L L D$ variation on Dead time values

By definition, ADC dead time is regarded as the total of the time intervals during which ADC has no ability for processing other events. Dead time can happen in all NDA system constituents. Dead time intervals are whether fixed or a function of system factors as well as pulse amplitude. Regarding MCA-based systems, dead time starts when the output pulse of amplifier crosses the threshold of ADC discriminator (Doug et al., 1991). As shown by each of the obtained results, the dead time is almost constant in all cases.

The NaI detector is operated by spectroscopic software (WinSpec software), which contains threshold function of image conversion into binary mode (black and white). Below the threshold setting, all intensity values are zero (black), while all those above turn to be ones (white) (Princeton Instruments WinSpes, 1999). The threshold value is also recorded in the spectrum, including multi-energy lines, and the net area is recorded. Changes in threshold values are not strongly affected by the net area of the photo peaks, as shown in Fig. 6 for radioactive point sources. The Figure shows that the net area is still constant in the range from $3 \%-6 \%$ for all photo peaks. 

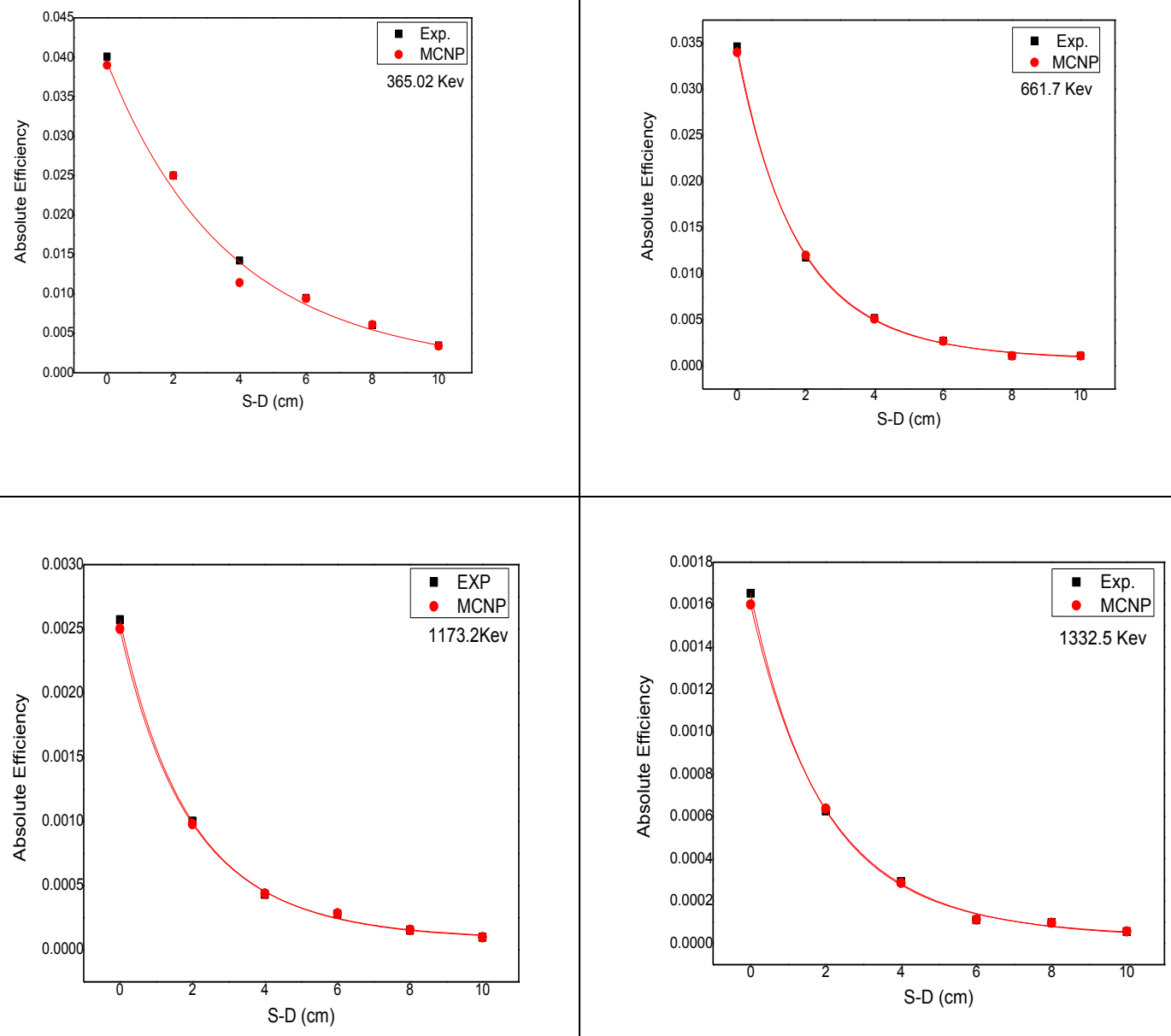

Fig. 4. The experimental results are matched to the calculated results using MCNP-5 code with negligible differences using ${ }^{133} \mathrm{Ba},{ }^{137} \mathrm{Cs}$ and ${ }^{60} \mathrm{Co}$.
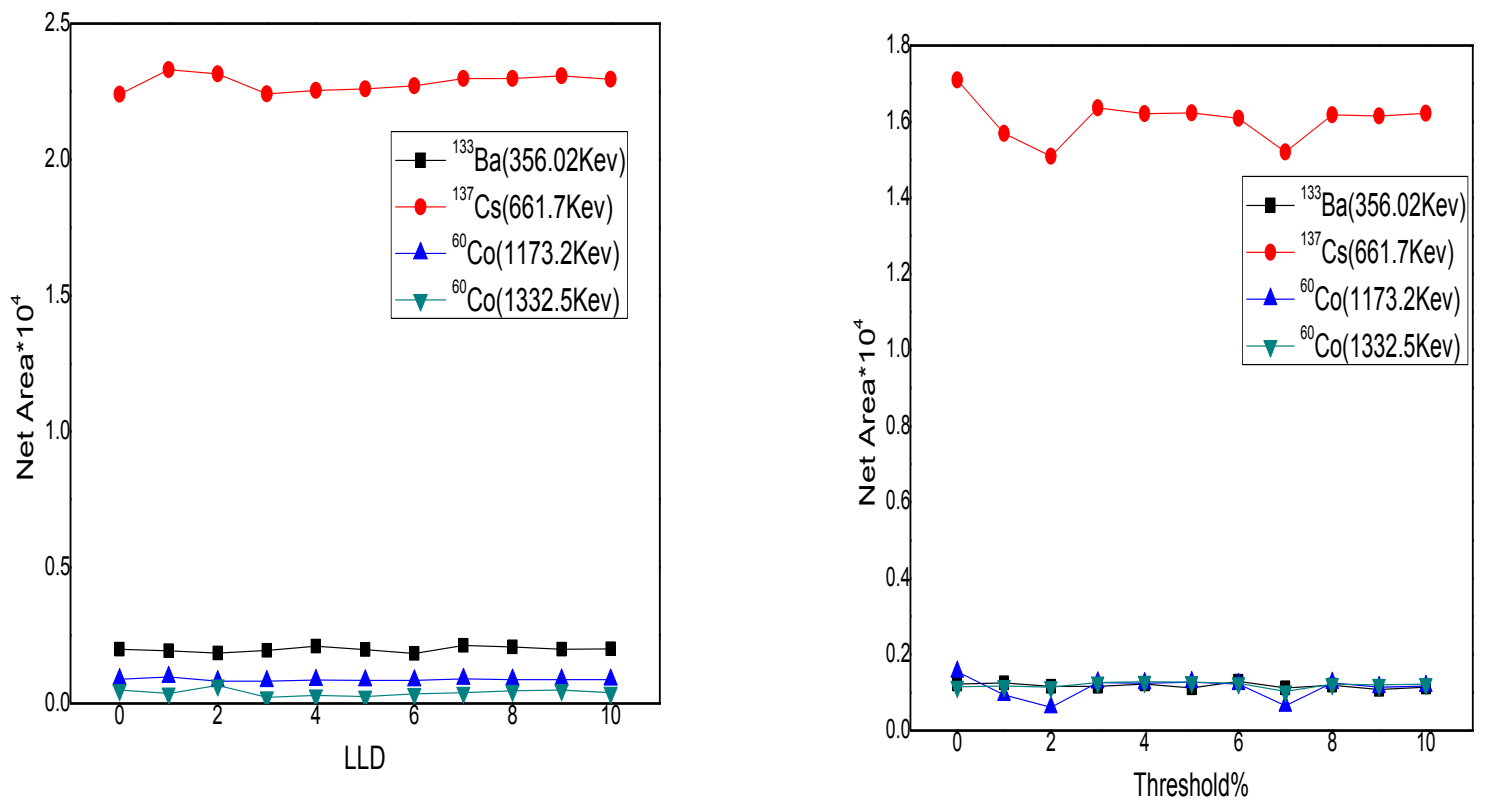

Fig. 5 The effect of LLD on the net area of the energy line Fig. 6. The effect of threshold on the net area for photo for point source ${ }^{133} \mathrm{Ba},{ }^{137} \mathrm{Cs},{ }^{60} \mathrm{Co}$. peaks of different point sources. 


\section{Conclusion}

A $\mathrm{NaI}(\mathrm{Tl})$ gamma detector was characterized with respect to FWHM, detector efficiency, lowlevel discriminator, and threshold effects using low, medium, and high-energy radioactive point sources. MCNP5 was used to simulate peak height distribution using GEB card by FWHM-value measurement at different energies in the spectra. The experimental results are strongly matched with those calculated using MCNP-5 code of ${ }^{133} \mathrm{Ba}$, ${ }^{137} \mathrm{Cs}$, and ${ }^{60} \mathrm{Co}$. The spectral data collected using inorganic scintillation detectors $(\mathrm{NaI}(\mathrm{Tl})$ showed that the variation of LLD values on the net area of photopeak affected the near stability in values of net area of the interested photopeak at energy lines of $356.017,661.7 .1173 .2$ and $1332.5 \mathrm{keV}$ starting from $L L D=4$ to 10 , while the dead-time values are almost constant in all energy regions. When threshold values have changed, the values of the net area are still constant in the range from $3 \%$ to $6 \%$ for all photo peaks. These parameters can enhance the performance of the measurement level in safeguards field.

\section{References}

Abdel-Monem, Y.K., Aladham, K.A., Sakr, H.A., Hazzaa, M.H., Eltybaney, R.A. (2019) Utilization of MCNP Code and ISOCS Software to Develop a Correlation For Comparative Measurements of Uranium Oxide Standards. J. Mater. Environ. Sci. 10(12), 1362-68.

Ahmadi, M., Rabbani, M., Mir Ahmadpour, P., (2009) Appropriate $\mathrm{NaI}(\mathrm{Tl})$ Detector for Measuring Gamma-Ray Emitted from ${ }^{137} \mathrm{Cs}$ in Sea Water Using Monte Carlo Code MCNP. 4C Simulation. J. Appl. Chem. Res. (JACR), 3(10), 55-60.

Ahmed, Z. (2017) Validation of gamma ray detector's model for safeguards application. Arab Journal of Nuclear Sciences and Applications, 50(4), 79-84.

Arnold, D., Sima, O. (2004) Extension of the efficiency calibration of germanium detectors using the GESPECOR software. Appl. Radiat. Isot. 61(2-3), $117-21$.

Ashrfi, S., Likar, A., Vidmar, T. (1999) Precise modeling of a coaxial HPGe detector.Nuclear Instruments and Methods A, 438(2-3), 421-28.

Avignone, III F.T. (1980) Monte Carlo code for predicting the response of a Ge\& $\mathrm{NaI}(\mathrm{T} 1)$ Compton supression spectrometer. Nuclear Instruments and Methods A, 174(3), 555-63.
De Castro, N.V., Le'vesque, R.J.A. (1967) Photopeak and double-escape peak efficiencies of germanium lithium drift detectors.Nuclear Instruments and Methods A, 46(2), 325-32.

Debertin, K., Grosswendt, B. (1982) Efficiency calibration of semiconductor detectors by primary standard sources and Monte Carlo calculations. Nuclear Instruments and Methods A, 203(1-3), 34352.

Doug, R., Norbert, E., Hastings, S., Jr. (1991) Passive Nondestructive Assay of Nuclear Materials, United State, Nuclear Regulatory Commission.

Eckert, Ziegler (2007) Certified Standard point sources, California-USA. Amersham, Gamma Reference Source Set (The Health Science Group), USA.

El-Gammal, W., El-Nagdy, M., Rizk, M., Shawky, S., Samei, M.A (2005) Verification of nuclear fuel plates by a developed non-destructive assay method, Nuclear Instruments and Methods A, 553, 627-38.

Ewa, I.O.B., Bodizs, D., Czifrus, Sz., Molnar, Zs., et al., (2001) Monte Carlo determination of full energy peak efficiency for a HPGe detector. Appl. Radiat. Isot. 55(1),103-08.

Fehrenbacher, G., Meckbach, R., Jacob, P. (1996) Unfolding the response of a Ge detector used for insitu gamma-ray spectrometry. Nuclear Instruments and Methods A, 383(2-3), 454-62.

Glenn, F., Knoll, (2010) "Radiation Detection and Measurement", $4{ }^{\text {th }}$ ed., United States of America.

Heath, R.L. (1997) Scientillation spectrometry GammaRay spectrum catalogue.

Hurtado, S., Garcia-Leon, M., Garcia-Tenorio, R., (2004a) GEANT4 code for simulation of a germanium gamma-ray detector and its application to efficiency calibration. Nuclear Instruments and Methods A, 518(3), 764-74.

Hurtado, S., Garcia-Leon, M., Garcia-Tenorio, R., (2004b) Monte Carlo simulation of the response of a germanium detector for low-level spectrometry measurements using GEANT4. Appl. Radiat. Isot. 61(2-3), 139-43.

Kadhem, A.B., Mohammed, A.N. (2010) The treatment of efficiency of $\mathrm{NaI}(\mathrm{Tl})$ detector by using Monte Carlo Simulation. Engineering and Technology Journal, 28(5), 1001-1013.

Kamboj, S., Kahn, B. (2003) Use of Monte Carlo simulation to examine gamma-ray interactions in 
germanium detectors. Radiat. Meas. 37(1), 1-8.

Kaneko, J., Katagiri, M., Ikeda, Y., Nishitani, T. (1998) Analysis on signals from synthetic diamond radiation detectors and suppression of a polarization phenomenon, Proc. 12 Workshop on Radiation Detectors and Their Uses, KEK, Tsukuba, pp. 25963.

Karamanis, D. (2003) Efficiency simulation of HPGe and $\mathrm{Si}(\mathrm{Li})$ detectors in $\gamma$ - and X-ray spectroscopy. Nuclear Instruments and Methods A, 505(1-2), 28285 .

Laborie, J.M., Le Petit, Abt, G.D., Girard, M. (2000) Monte Carlo calculation of the efficiency calibration curve and coincidence-summing corrections in lowlevel gamma-ray spectrometry using well-type HPGe detectors. Appl. Radiat. Isot. 53(1-2), 57- 62.

Landau, D.P., Binder, K. (2009) "A Guide to Monte Carlo Simulations in Statistical Physics", $3^{\text {rd }}$ ed., New York: Cambridge University Press, xv, pp. 471, ISBN: 978-0-521-76848-1.

Lepy M.C., Altzitzoglou, T., Arnold, D., et al. (2001) Intercomparison of efficiency transfer software for gamma-ray spectrometry. Appl. Radiat. Isot. 55(4), 493-503.

Lodge, M.A., Holt, D.P., Kinahan, P.E., Wong, D.F., Wahl, R.L. (2015) Performance assessment of a $\mathrm{NaI}(\mathrm{Tl})$ gamma counter for PET applications with methods for improved quantitative accuracy and greater standardization. EJNMMI Physics, 2, 11.

Meixne, C. (1974) A Monte Carlo program for the calculation of gamma-ray spectra for germanium detectors. Nuclear Instruments and Methods A, 119, 521-26.

Michel, C., Emling, H., Grosse, E., Azgui, F., Grein, H., Wollersheim, H.J., Gaardhoje, J.J., Herskind, B. (1986) Monte Carlo simulation of complex germanium detector systems and Compton suppression spectrometers. Nuclear Instruments and Methods A, 251(1),119-33.

Nakazawa, D., Bronson, F., Croft, S., McElroy, R., Mueller, W.F., Venkataraman, R. (2010) The Efficiency Calibration of Non-Destructive Gamma Assay Systems Using Semi-Analytical Mathematical Approaches, 10497, WM2010 Conference, March 7-11, , Phoenix, AZ.
Princeton Instruments WinSpes (1999) Princeton Instruments, spectroscopic software, Version 2.4.M, September 20 .

Rodenas, J., Martinavarro, A., Rius, V. (2000) Validation of the MCNP code for the simulation of Ge-detector calibration. Nuclear Instruments and Methods A, 450(1), 88-97.

Saegusa, J., Oishi, T., Kawasaki, K., Yoshizawa, M., Yoshida, M., Sawahata, T., Honda, T. (2000) Determination of gamma-ray efficiency curves for volume samples by the combination of Monte Carlo simulations and point source calibration. J. Nucl. Sci. Technol. 37(12), 1075-81.

Salgado, C.M., Conti, C.C., Becker, P.H.B., (2006) Determination of $\mathrm{HPGe}$ detector response using MCNP5 for 20-150 keV X-rays. Appl. Radiat. Isot. 64(6), 700-05.

Savidou, A., Tzika, F., Stamatelatos, I.E. (2008) Further Development in Characterization of Radioactive Waste Drums by Non Destructive Gamma Spectrometry at GRR-1, Institute of Nuclear Technology and Radiation Protection, NCSR "Demokritos", Aghia Paraskevi, Greece.

Tarim, U.A., Gurler, O. (2018) Source-to-detector Distance Dependence of Efficiency and Energy Resolution of a 3"x3" NaI(Tl) Detector. European Journal of Science and Technology, 13, 103-107.

Unz, R.J., Rogers, D.M., Waggoner, Ch.A. (2011) Characterizing Inorganic Scintillation Detectors for Determining Radiation Exposure, 11358, WM2011 Conference, February 27 - March 3, Phoenix, AZ.

Varley, B.J., Kitching, J.E., Leo, W., Miskin, J., Moore, R.B., Wunsch, K.D., Decker, R., Wollnik H., Siegert G. (1981) Investigations of the response of germanium detectors to monoenergetic electron, positron and gamma ray beams. Nuclear Instruments and Methods A, 190(3), 543-54.

Wainio, K.M., Knoll, G.F., (1966) Calculated gamma ray response characteristics of semiconductor detectors. Nuclear Instruments and Methods A, 44(2), 213-23.

X-5 Monte Carlo Team, (2003) MCNP - A General Monte-Carlo N-Particle Trans port Code, Version 5, LA-UR-03-1987. 